IJMMS 29:3 (2002) 179-181

PII. S0161171202007706

http://ijmms.hindawi.com

(c) Hindawi Publishing Corp.

\title{
AN APPLICATION TO KATO’S SQUARE ROOT PROBLEM
}

\author{
TOKA DIAGANA
}

Received 24 May 2001

We find all complex potentials $Q$ such that the general Schrödinger operator on $\mathbb{R}^{n}$, given by $L=-\Delta+Q$, where $\Delta$ is the Laplace differential operator, verifies the well-known Kato's square problem. As an application, we will consider the case where $Q \in L_{\mathrm{loc}}^{1}(\Omega)$.

2000 Mathematics Subject Classification: 47B44, 35J05.

1. Introduction. Let $\Omega \subseteq \mathbb{R}^{n}$, an open set and let's stand in the Hilbert space $H=$ $L^{2}(\Omega, C)\left(=L^{2}(\Omega)\right)$. Consider $Q$, a measurable complex function and let $\Phi$ and $\Psi$ be the sesquilinear forms given by,

$$
\begin{aligned}
& \Phi(u, v)=\int_{\Omega} \nabla u \overline{\nabla v} d x \quad \forall u, v \in D(\phi)=H_{0}^{1}(\Omega), \\
& \Psi(u, v)=\int_{\Omega} Q u \bar{v} d x \quad \forall u, v \in D(\Psi),
\end{aligned}
$$

where $D(\Psi)=\left\{u \in L^{2}(\Omega): Q|u|^{2} \in L^{1}(\Omega)\right\}$. Assume that the potential $Q$ verifies that there exists $\beta>0$ and there exists $\theta \in(0, \pi / 2)$, such that

$$
|\arg (Q-\beta)| \leq \frac{\pi}{2}-\theta .
$$

The sesquilinear forms $\Phi$ and $\Psi$ are both closed, densely defined, and sectorial. According to Kato's first representation theorem (see [2]), we can associate to $\Phi$ and $\Psi, m$-sectorial linear operators defined, respectively, by

$$
\begin{array}{lll}
A u=-\Delta u & \text { with } & D(A)=\left\{u \in H_{0}^{1}(\Omega): \Delta u \in L^{2}(\Omega)\right\}, \\
B u=Q u & \text { with } & D(B)=\left\{u \in L^{2}(\Omega): Q u \in L^{2}(\Omega)\right\} .
\end{array}
$$

By Schrödinger operator, we mean a partial differential operator on $\mathbb{R}^{n}$ of the form

$$
L=A+B ; \quad A=-\Delta ; \quad B=Q=Q(x),
$$

where $\Delta$ is the $n$-dimensional Laplace operator $\Delta=\sum_{i=1}^{n} \partial^{2} / \partial x_{i}^{2}$. The name comes from the form of Schrödinger's equation which, in units with $h=m=1$ reads

$$
i \frac{\partial u}{\partial t}=L u \text {. }
$$

Our aim here is to find all potentials $Q$ such that

$$
D\left(L^{1 / 2}\right)=D\left(A^{1 / 2}\right) \cap D\left(B^{1 / 2}\right)=D\left(L^{* 1 / 2}\right) .
$$


For that we use the author results [1] related to the sum of linear operators connected to Kato's square root problem. The case where $\Omega=\mathbb{R}^{n}$ will be studied later as a consequence of the general case.

\section{Schrödinger operators and Kato's condition}

DEFINITION 2.1. A linear operator $C$ is said to verify Kato's square root problem (or Kato's condition) if

$$
D\left(C^{1 / 2}\right)=D(Y)=D\left(C^{* 1 / 2}\right)
$$

where $Y$ is the sesquilinear form associated to $C$.

Hypothesis on $Q$. Suppose that $Q$ is chosen such as,

$$
\overline{D(\Phi) \cap D(\Psi)}=L^{2}(\Omega)
$$

Proposition 2.2. Let $A$ and $B$ be the linear operators given by (1.3). Assume that the potential $Q$ verifies (2.2). Then there exists a unique operator sum $A \oplus B$, which is $m$-sectorial, verifying Kato's condition and

(i) $A \oplus B=\overline{A+B}$ if $\overline{A+B}$ is a maximal operator,

(ii) $|\operatorname{Im}\langle(A \oplus B) u, u\rangle| \leq \operatorname{Re}\langle(A \oplus B) u$, $u\rangle$, for all $u \in D(A \oplus B)$.

Proof. Assume that $Q$ verifies hypothesis (2.2). So, the sesquilinear form given by, $Y=\Phi+\Psi$, is a closed, sectorial, and densely defined. By Kato's first representation theorem (see [2]), there exists a unique $m$-sectorial sum operator, $A \oplus B$, associated to , verifying

$$
\Upsilon(u, v)=\langle(A \oplus B) u, v\rangle \quad \forall u \in D(A \oplus B), v \in D(\Phi) \cap D(\Psi)
$$

since $A$ and $B$ both verify Kato's condition, according to author's theorem (see [1, Theorem 2, page 462]), the operator $A \oplus B$ verifies the same condition, that is,

$$
D\left((A \oplus B)^{1 / 2}\right)=D(\Phi) \cap D(\Psi)=D\left((A \oplus B)^{* 1 / 2}\right)
$$

and (ii) is satisfied, where $\beta$ is given by (1.2). The operator $A \oplus B$ is defined as

$$
\begin{aligned}
& (A \oplus B) u=-\Delta u+Q u, \quad \forall u \in D(A \oplus B), \\
& D(A \oplus B)=\left\{u \in H_{0}^{1}(\Omega): Q|u|^{2} \in L^{1}(\Omega),-\Delta u+Q u \in L^{2}(\Omega)\right\} .
\end{aligned}
$$

Using also author's theorem (see [1, Theorem 2, page 462]), it follows that (i) is satisfied.

3. Some applications. Consider the same operators, that is, $A=-\Delta$ and $B=Q$ in $L^{2}(\Omega)$. Assume that $Q \in L_{\mathrm{loc}}^{1}(\Omega)$, in this case (2.2) is satisfied. According to Brézis and Kato, the operator $\overline{A+B}$ is maximal in $L^{2}(\Omega)$ (then $A \oplus B=\overline{A+B}$ and Kato's condition is satisfied) and is given by (2.5). 
CASE $\Omega=\mathbb{R}^{n}$. We always assume $Q \in L_{\text {loc }}^{1}\left(\mathbb{R}^{n}\right)$, it follows that

$$
\begin{aligned}
& A u=-\Delta u \quad \text { with } \quad D(A)=H^{2}\left(\mathbb{R}^{n}\right) ; \quad D\left(A^{1 / 2}\right)=H^{1}\left(\mathbb{R}^{n}\right), \\
& B u=Q u \quad \text { with } \quad D\left(B^{1 / 2}\right)=\left\{u \in L^{2}\left(\mathbb{R}^{n}\right): Q|u|^{2} \in L^{1}\left(\mathbb{R}^{n}\right)\right\},
\end{aligned}
$$

and $D\left(A^{1 / 2}\right) \cap D\left(B^{1 / 2}\right)$ is dense in $L^{2}\left(\mathbb{R}^{n}\right)$ because,

$$
C_{0}^{\infty}\left(\mathbb{R}^{n}\right) \subseteq D(\Phi) \cap D(\Psi) .
$$

In conclusion, Kato's condition is satisfied by $\overline{A+B}$, that is,

$$
D(\sqrt{\overline{A+B}})=D(\sqrt{A}) \cap D(\sqrt{B})=D\left(\sqrt{\overline{A+B}}{ }^{*}\right) .
$$

For example when $n=1$, then

$$
D(\sqrt{\overline{A+B}})=H^{1}(\mathbb{R})=D\left(\sqrt{\overline{A+B}^{*}}\right) .
$$

REMARK 3.1. Condition (2.2) could be weakened as

$$
\overline{D(A) \cap D(B)}=L^{2}(\Omega) .
$$

But in general the algebraic sum of two operators is not always defined (because this concept is not well adapted to problems arising in mathematical analysis).

ACKNOWLedgement. I would like to express my thanks to Prof. Hofmann about our discussions on this note.

\section{REFERENCES}

[1] T. Diagana, Sommes d'opérateurs et conjecture de Kato-McIntosh [Sums of linear operators and the Kato-McIntosh conjecture], C. R. Acad. Sci. Paris Sér. I Math. 330 (2000), no. 6, 461-464 (French).

[2] T. Kato, Perturbation Theory for Linear Operators, Die Grundlehren der mathematischen Wissenschaften, vol. 132, Springer-Verlag, New York, 1966.

TOKA Diagana: Department OF MATHEMATICS, Howard UNIVERSity, 2441, SiXTH STREET, NW WASHINGTON, DC 20059, USA

E-mail address: tdi agana@howard.edu 


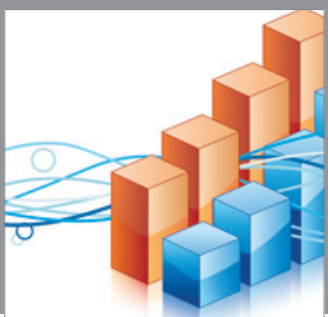

Advances in

Operations Research

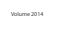

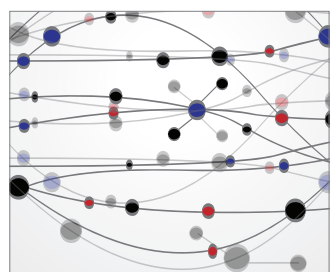

\section{The Scientific} World Journal
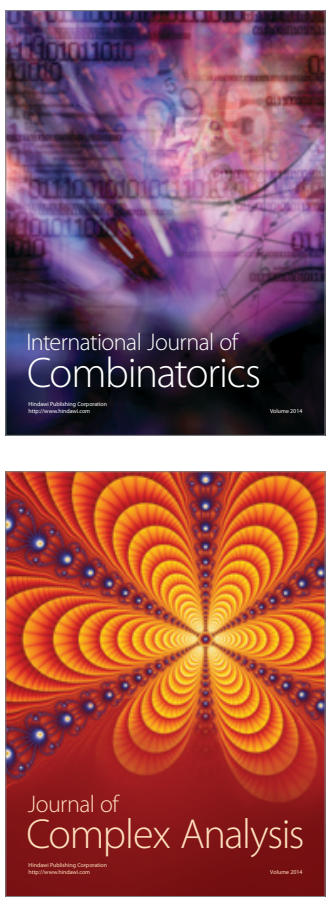

International Journal of

Mathematics and

Mathematical

Sciences
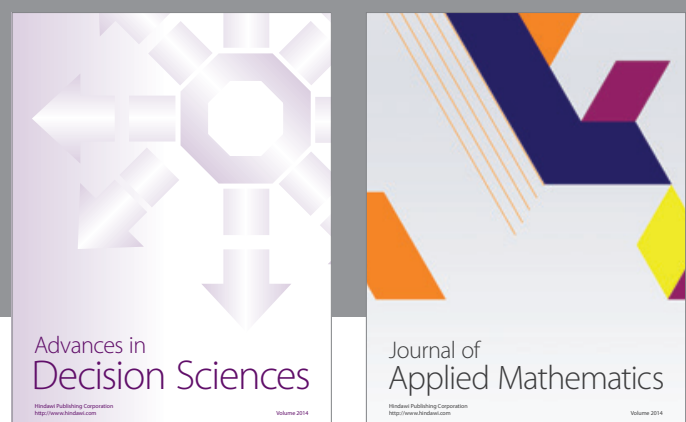

Journal of

Applied Mathematics
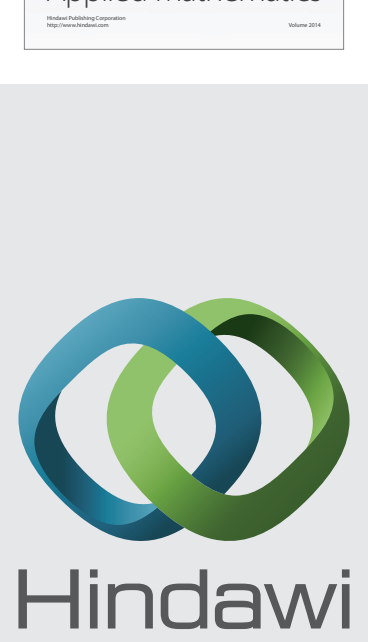

Submit your manuscripts at http://www.hindawi.com
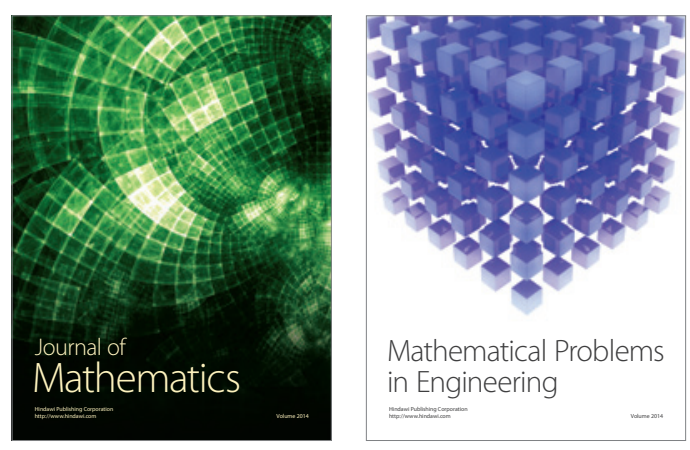

Mathematical Problems in Engineering
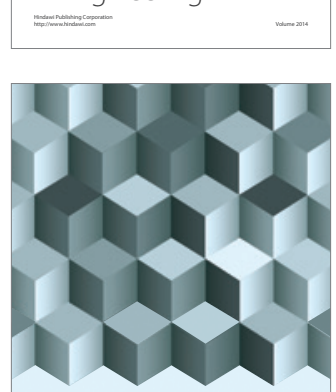

Journal of

Function Spaces
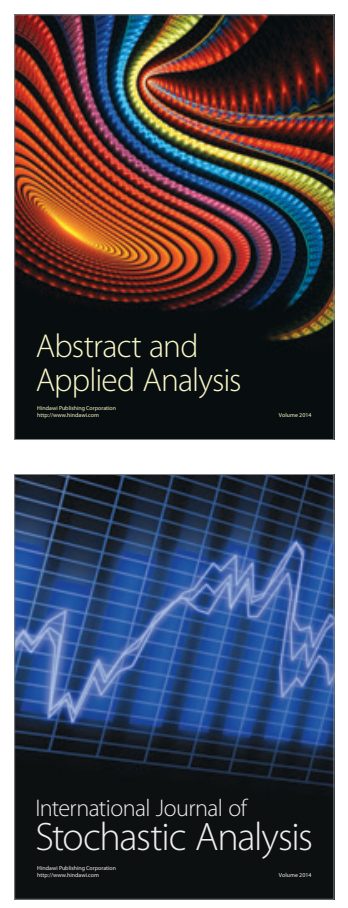

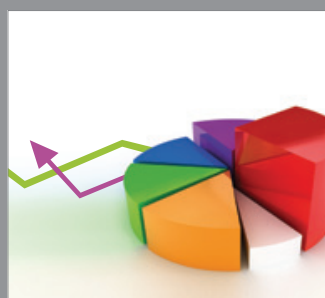

ournal of

Probability and Statistics

Promensencen
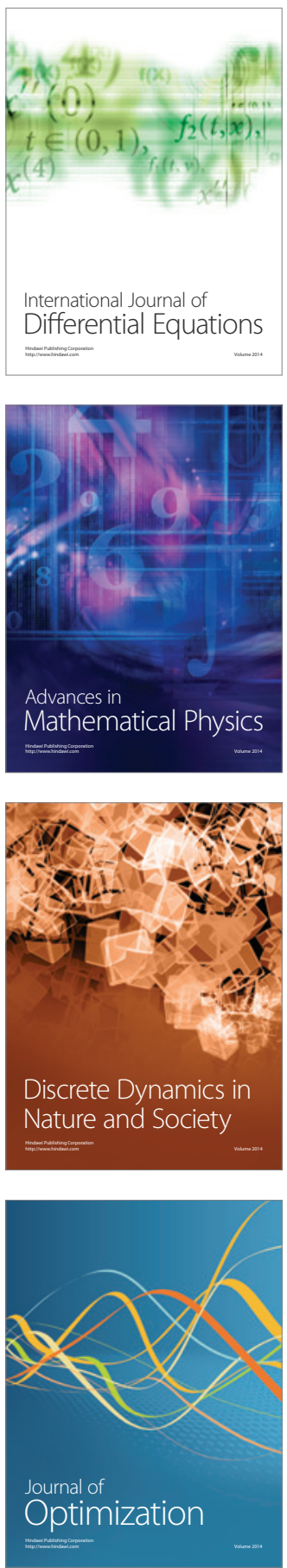